\title{
Effects of therapeutic hypothermia on white matter injury from murine neonatal hypoxia-ischemia
}

\author{
Elliot Koo ${ }^{1}$, R. Ann Sheldon ${ }^{1}$, Byong Sop Lee ${ }^{2}$, Zinaida S. Vexler ${ }^{3}$ and Donna M. Ferriero ${ }^{1}$
}

BACKGROUND: Therapeutic hypothermia (TH) is the standard of care for neonates with hypoxic-ischemic encephalopathy, but it is not fully protective in the clinical setting. Hypoxia-ischemia (HI) may cause white matter injury (WMI), leading to neurological and cognitive dysfunction.

METHODS: P9 mice were subjected to $\mathrm{HI}$ as previously described. Pups underwent $3.5 \mathrm{~h}$ of systemic hypothermia or normothermia. Cresyl violet and Perl's iron staining for histopathological scoring of brain sections was completed blindly on all brains. Immunocytochemical (ICC) staining for myelin basic protein (MBP), microglia (Iba1), and astrocytes (glia fibrillary acidic protein (GFAP)) was performed on adjacent sections. Volumetric measurements of MBP coverage were used for quantitative analysis of white matter.

RESULTS: TH provided neuroprotection by injury scoring for the entire group $(n=44 ; P<0.0002)$. ICC analysis of a subset of brains showed that the lateral caudate was protected from WMI $(P<0.05)$. Analysis revealed decreased GFAP and Ibal staining in hippocampal regions, mostly CA2/CA3. GFAP and Iba1 directly correlated with injury scores of normothermic brains.

CONCLUSION: TH reduced injury, and qualitative data suggest that hippocampus and lateral caudate are protected from HI. Mildly injured brains may better show the benefits of $\mathrm{TH}$. Overall, these data indicate regional differences in WMI susceptibility and inflammation in a P9 murine $\mathrm{HI}$ model.

$\mathbf{N}$ eonatal hypoxia-ischemia (HI) treated with hypothermia has become a standard of care for infants. Hypoxicischemic encephalopathy occurs in three to five per 1,000 live births and produces a high morbidity rate and severe/longterm neurological and cognitive deficits with high outcomes in cerebral palsy, epilepsy, and mental retardation (1). However, no therapy completely protects against neonatal $\mathrm{HI}$ encephalopathy; therefore, opportunities exist to continue to explore additional agents (2). Therapeutic hypothermia (TH) has been shown to decrease the mortality rate and display neuroprotection in infants with neonatal HI encephalopathy (3-5). There have been many mechanisms implicated in the effects of hypothermic therapy, which include the suppression of apoptosis, decreased inflammation, reduction in excitatory amino acids, decreased reactive oxygen species, and a reduced cerebral metabolic rate (6).

A previous study using $\mathrm{P} 7$ rats investigating the use of hypothermia and erythropoietin suggested that slightly older animals that are modeled equivalent to the full-term newborn brain may be necessary to observe the benefits of adjuvant therapy (7). In addition, there has been evidence that $\mathrm{TH}$ displays neuroprotection for specific regions of the brain, such as the hippocampus and thalamus (8). Interestingly, the results of some studies show that mildly injured brains may receive the benefits of $\mathrm{TH}$ relating to white matter injury (WMI) and overall neuroprotection, whereas severely injured brains do not. This trend of insufficient benefit from TH for severely injured brains is consistent with the findings in other animal models (9-11).

The presence of white matter (WM) and appropriate axon myelination is essential to normal brain connectivity and development in the newborn infant (12). It has been found that insufficient myelination leads to neurological and cognitive dysfunction $(13,14)$. Little investigation has been done on the effects of hypothermia on WMI. There is evidence showing that mild hypoxemia in neonatal mice causes WM hypomyelination and WMI (15). Rodent studies of HI have shown that the developmental stage of the brain, preterm vs. term, at the time of injury has a major role in the underlying WMI, partly because of a maturation-dependent oligodendrocyte arrest (16).

TH neuroprotection from WMI in a full-term murine model for HI has described the evolution of brain injury (17). A previous TH study in sheep displayed neuroprotection from WMI (18). The hypothesis tested is that TH will reduce WMI in P9 neonatal mice subjected to a $\mathrm{HI}$ insult through reduction of the glial/inflammatory response. The hypothesis is addressed by evaluating the extent of these processes by volumetric analysis and immunocytochemical assessments.

\section{METHODS}

\section{Hypoxia-Ischemia}

P9 male and female CD1 mice were subjected to focal ischemia with global hypoxia, as previously described in the Rice-Vannucci model (19). All procedures were approved by the Institutional Animal Care and Use Committee at University of California San Francisco, in

\footnotetext{
'Department of Pediatrics, University of California San Francisco, San Francisco, California; ${ }^{2}$ Department of Neurology, University of Ulsan College of Medicine, Seoul, Korea; ${ }^{3}$ Department of Neurology, University of California San Francisco, San Francisco, California. Correspondence: Donna M. Ferriero (Donna.Ferriero@ucsf.edu) 
accordance with National Institutes of Health guidelines for the Care and Use of Laboratory Animals. Ligation of the left common carotid artery was carried out. Mice were anesthetized with 3\% isoflurane, balance oxygen. A midline incision of the neck was done to dissect and ligate the left common carotid artery via electrical coagulation. Pups were returned to the dam for $1 \mathrm{~h}$ following the procedure. Hypoxia was induced by placing pups in chambers submerged in a $37-{ }^{\circ} \mathrm{C}$ water bath into which an atmosphere of $10 \%$ oxygen and $90 \%$ nitrogen was introduced via inlet and outlet tubing (maintained by a flow meter). Pups were removed from chambers after $50 \mathrm{~min}$ and returned to the dam for $1 \mathrm{~h}$.

\section{Hypothermia}

The pups were then equally separated into chambers maintained at either $30^{\circ} \mathrm{C}(n=30)$ or $36.5^{\circ} \mathrm{C}(n=24)$, with both chambers open to room air, for $3.5 \mathrm{~h}$ of either HT or NT. One sentinel cooled mouse was monitored with a temperature probe. All pups also had their temperature monitored with an infrared thermometer (Supplementary Figure S1). Following cooling, the temperature in the chambers of the HT pups was gradually increased to $37^{\circ} \mathrm{C}$ over $30 \mathrm{~min}$.

\section{Histology}

For histopathological examination, pups were killed at P14. A lethal dose of pentobarbital was used for anesthesia before transcardiac perfusion with ice-cold $4 \%$ paraformaldehyde in $0.1 \mathrm{M}$ phosphatebuffered saline. The brains were removed and postfixed overnight in the same solution for $4 \mathrm{~h}$, and then equilibrated in $30 \%$ sucrose in $0.1 \mathrm{M}$ phosphate-buffered saline. A majority of the brains $(n=40)$ were cut on a Vibratome, and sequential sections (50 um) were collected for Nissl (cresyl violet) and Perl's iron staining. The remaining brains ( $n=14,3$ sham brains not analyzed) were frozen, cut on a cryostat (Leica Microsystems, Wetzlar, Germany), and 20$\mu \mathrm{m}$ coronal sections were collected on Superfrost slides and stored at $-80^{\circ} \mathrm{C}$. Following sectioning, samples were stained with cresyl violet (Nissl stain), dehydrated in graded ethanol solutions, cleared in Citrisolv (Fisher Scientific, Hampton, NH), and cover-slipped in Permount (Fisher Scientific).

Both cresyl-violet- and Perl's iron-stained sections were used for histopathological scoring of brain sections, which was completed in a blinded manner. Sections were assessed rostral to caudal. Regions scored included the following: anterior cortex; middle cortex; posterior cortex; caudate/striatum; hippocampus CA1, CA2, and CA3; and the dentate gyrus. Scores were assessed for each region as follows: $0=$ no injury, $1=$ minimal cell loss manifested by scattered shrunken neurons and glia with small patches of iron deposition, $2=$ moderate cell loss with infarction in a columnar distribution in the cortex with concomitant gliosis or the shrunken hippocampus with cell loss throughout the Sommer's sector with corresponding iron deposition, and $3=$ severe cell loss and gliosis with cystic infarction, for a total score of $0-24$. Injury scoring 5 days after HI was completed on P9 mice treated with either normothermia or therapeutic hypothermia (TH), using cresyl-violet- and Perl's ironstained adjacent sections (Supplementary Figure S2). The specimens that were used only for ICC staining were also scored.

\section{Immunofluorescence and Image Capturing}

To identify and quantify different cell types, two to four sections per region were blocked with $25 \%$ goat serum in PBST $(0.1 \%$ Triton$\mathrm{X}-100)+5 \%$ bovine serum albumin, followed by quadruple staining with primary antibodies. Primary antibodies were diluted in blocking solution as follows: Iba1 (microglia) at a ratio of 1:200 (Wako Chemicals, Richmond, VA); MBP at a ratio of 1:1,000 (Abcam, Cambridge, MA); and GFAP at a ratio of 1:2,000 (Thermo Fisher Scientific, Rockford, IL). Slides were incubated with primary antibodies overnight at $4{ }^{\circ} \mathrm{C}$, followed by washing and incubation with species-specific secondary antibodies that were applied at a dilution of 1:200: Alexa Fluor 568, 488, 647, and 350 (all Life Technologies, Eugene, OR). A final washing was carried out, and then

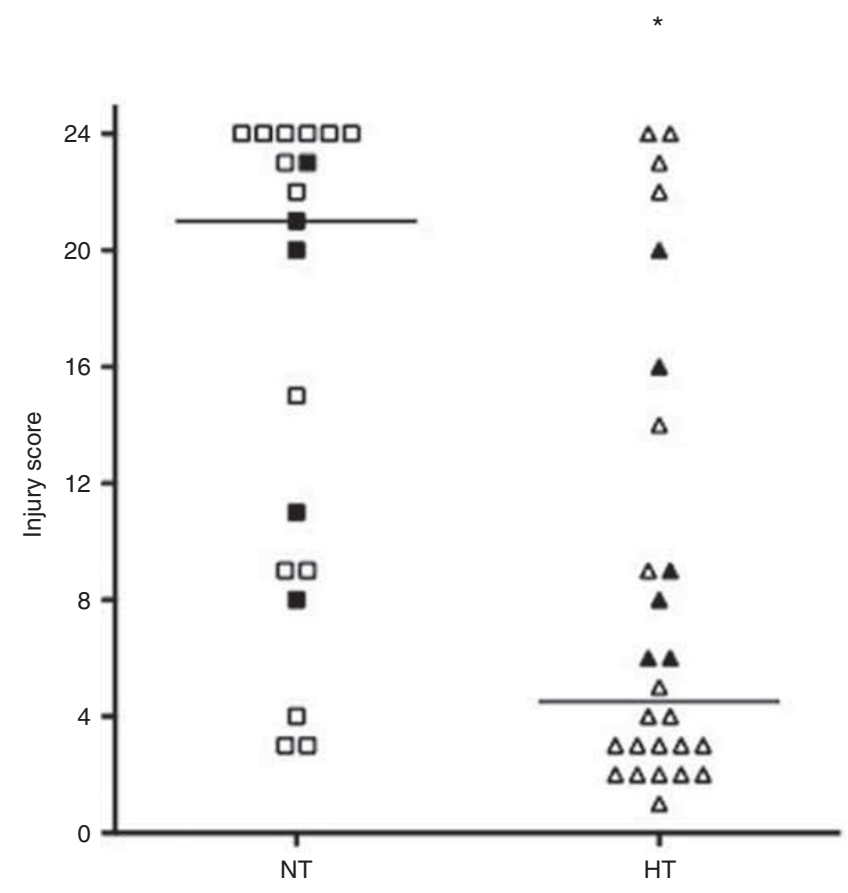

Figure 1. Histopathological analysis injury score of brain samples. Filled data points indicate samples that were also used for ICC analysis. Cresyl violet staining of brain sections for histopathological scoring of the ipsilateral hemispheres was completed blindly of all samples. Sections were scored rostral to caudal at anterior cortex, middle cortex, posterior cortex, caudate/striatum, hippocampus $\mathrm{CA} 1, \mathrm{CA} 2$, and $\mathrm{CA} 3$, and dentate gyrus. Scoring was performed according to the protocol by Sheldon et al. $0=$ no injury; $1=$ minimal cell loss, scattered shrunken neurons, and glia; 2 =moderate cell loss; $3=$ severe cell loss and gliosis with cystic infarction. Samples were compared according to the treatment group: normothermia and hypothermia.

the slides were mounted with a coverslip using the ProLong Gold antifade mountant (Life Technologies).

Image capturing and analysis were completed in a blinded manner using the Volocity Software-a confocal-like laser-free technology. For data analysis, $z$-stacks of stained sections were captured at $5-\mu \mathrm{m}$ intervals at $\times 10$ (Zeiss Axiovert 100 equipped with Openlab Software, Improvision, Coventry, UK). Captured images focused anatomically on the lateral caudate (which included a section of corpus callosum), medial caudate, hippocampus CA1 with dentate gyrus, and hippocampal regions CA2 and CA3. Volume coverage was analyzed for WM (MBP), astroglia (GFAP), and microglia (Iba1) using an automated protocol for a signal intensity threshold (1.5-2.0 SD background in channels) for the caudate (position 336-353) and the hippocampus (position 235-270) according to the Allen Brain Atlas of P14 brain strain C57BL/6J.

\section{Statistical Analysis}

Injury scores between normothermic and hypothermic groups were compared with the Mann-Whitney test. The analysis was performed with Prism 6.0 (GraphPad Software, La Jolla, CA).

Regional data for the summation of volumes of MBP, GFAP, and Ibal were collected and normalized using the ratio of intact ipsilateral tissue to intact contralateral hemisphere tissue. Data comparing HI treated with normothermia and HI treated with hypothermia were conducted using an unpaired $t$-test to determine statistical significance.

Data were compared according to the degree of injury ( $\operatorname{mild}=0-8$; moderate $=8-16$; and severe $=16-24)$. The raw volumetric data of MBP, GFAP, and Ibal were plotted against injury score of 


\section{Articles | Koo et al.}

a
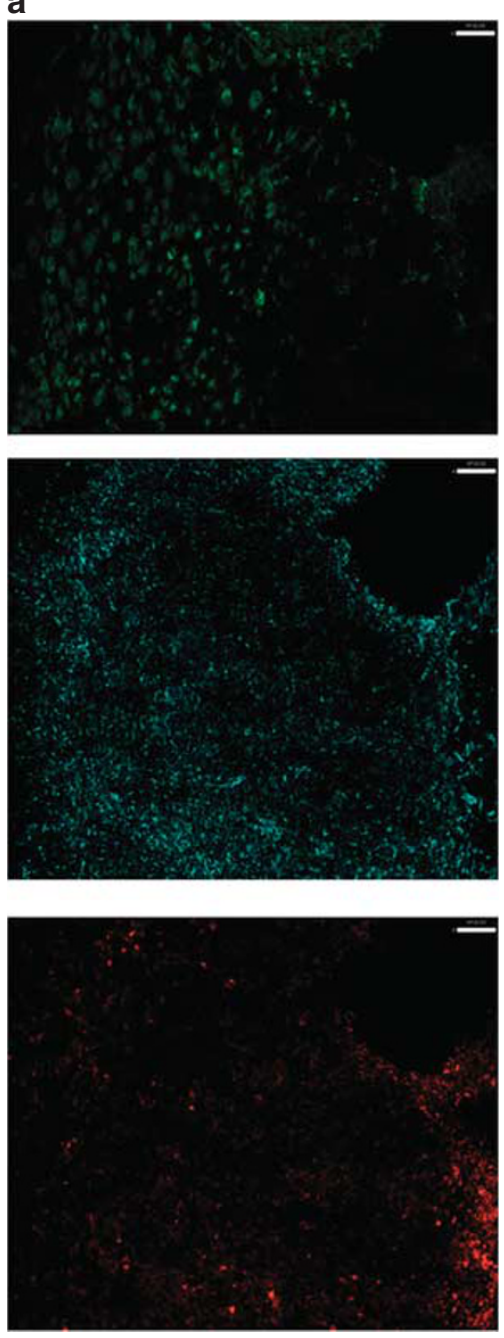

b
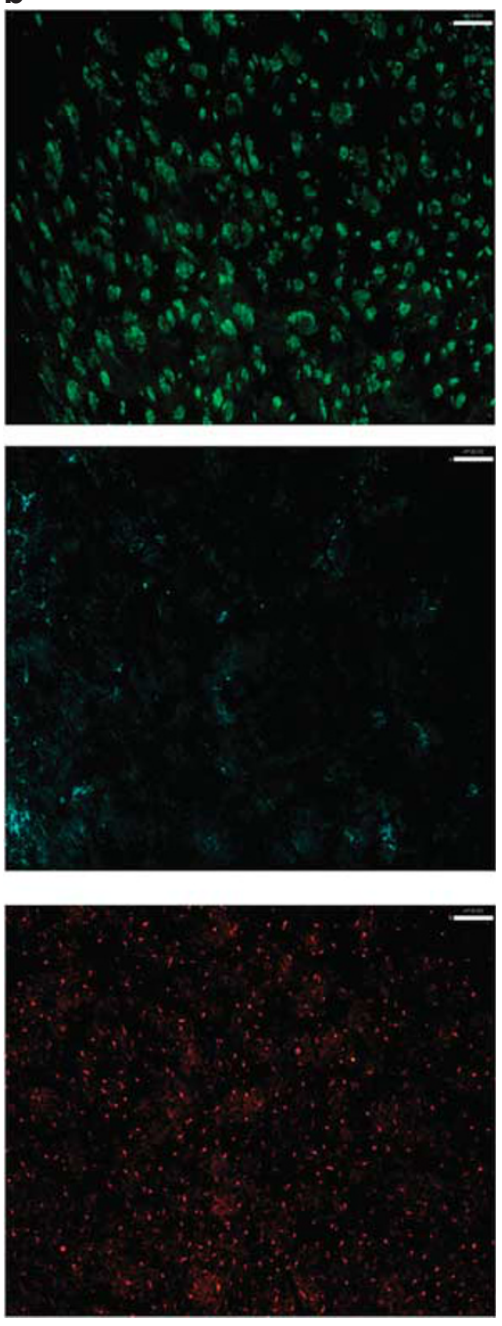

C

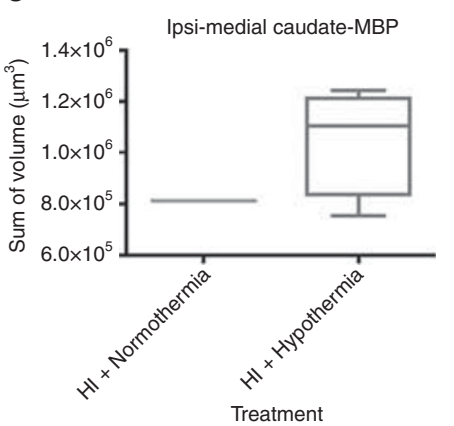

Ipsi-medial caudate-GFAP
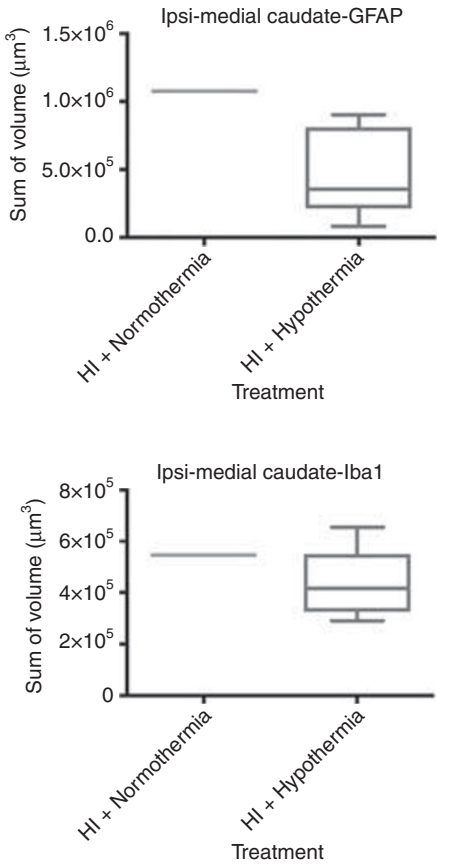

Figure 2. MBP, GFAP, and Iba1 coverage of the medial caudate. MBP (green), GFAP (far red), and Iba1 (red). Column (a) contains samples treated with $\mathrm{HI}+$ normothermia. Column (b) contains samples treated with HI+hypothermia. (c) Volumetric measurements normalized using the ratio of intact ipsilateral tissue to intact contralateral hemisphere tissue. Data comparing $\mathrm{HI}$ treated with normothermia and $\mathrm{HI}$ treated with hypothermia using a box-and-whisker graph. The horizontal line indicates the median score. Magnification is $\times 10$. Scale bar $=100 \mu \mathrm{m}$.

normothermic and hypothermic brain's caudate and hippocampal regions. Data collected from the medial and lateral caudate were combined, and the data from the hippocampus DG, CA1, CA2, and CA3 were combined. Correlation coefficients were calculated using the Pearson correlation coefficients test to determine the relationship between injury score with inflammation and WMI.

\section{RESULTS}

\section{Histopathological Analysis}

The analysis of all available brain samples showed that $\mathrm{TH}$ provides neuroprotection, as determined by injury scoring (Figure 1). The protection was seen in all regions analyzed: cortex $(P=0.0002)$; hippocampus $(P=0.0006)$; and caudate $(P=0.01)$. There were no sex differences in injury severity: normothermia-treated (NT) male vs. NT female $(n=10$ each), $P=0.16$; hypothermia-treated (HT) male $(n=13)$ vs. HT female $(n=12), P=1.0$. There was also no difference in incidence of mortality between groups: four NT and five HT died, $P=1.0$ by Fisher's exact test.

\section{Volumetric Immunocytochemistry Analysis}

ICC volumetric analysis showed that, compared with normothermic brains (Figures 2a and 3a), WMI was less in the ipsilateral caudate following $\mathrm{TH}$ (Figures $\mathbf{2} \mathbf{b}$ and $\mathbf{3 b}$ ). The lateral caudate was significantly protected from WMI $(P<0.05)$ with TH (Figure 3c), but there were no significant differences in glia fibrillary acidic protein (GFAP) or Iba1 volume. Volumetric analysis of the medial caudate showed that $\mathrm{TH}$ provides a wide range of protection from WMI, and although significance could not be determined from quantitative imaging data smaller MBP volumes suggest that $\mathrm{TH}$ plays a role in protection from WMI in the medial caudate (Figure 2c). Further, the medial caudate qualitative data of GFAP and Ibal (Figure 2a-c) also show that $\mathrm{TH}$ provides observable differences in imaging data compared with normothermia. Data collected from the hippocampal region CA1 and dentate gyrus showed no differences in MBP, GFAP, or Ibal volumes between normothermia-and TH-treated 
a
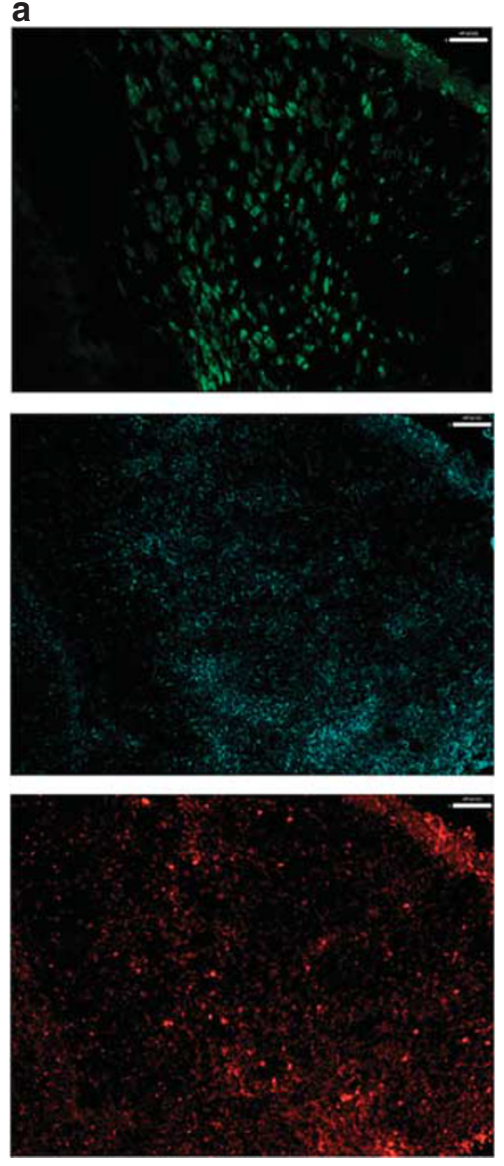

b
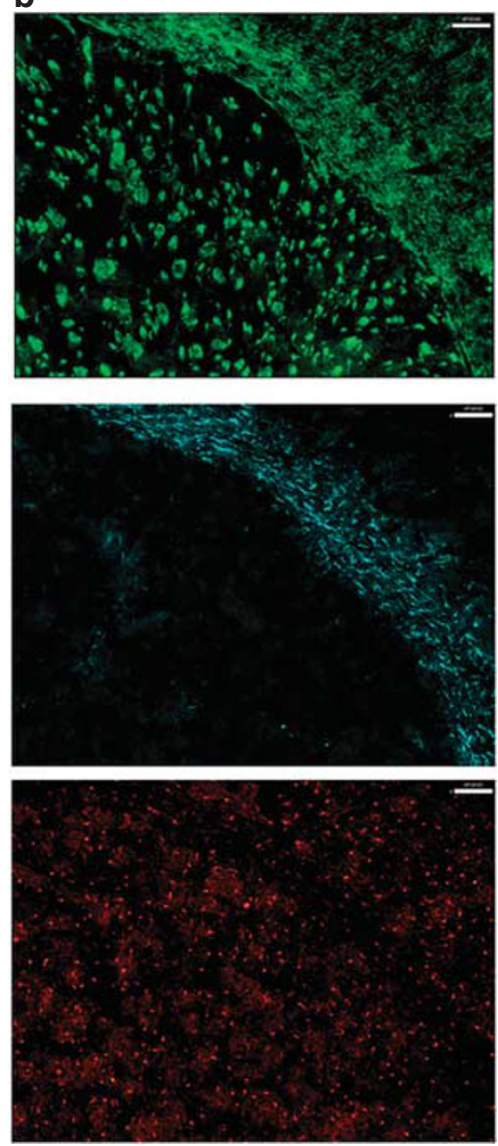

C
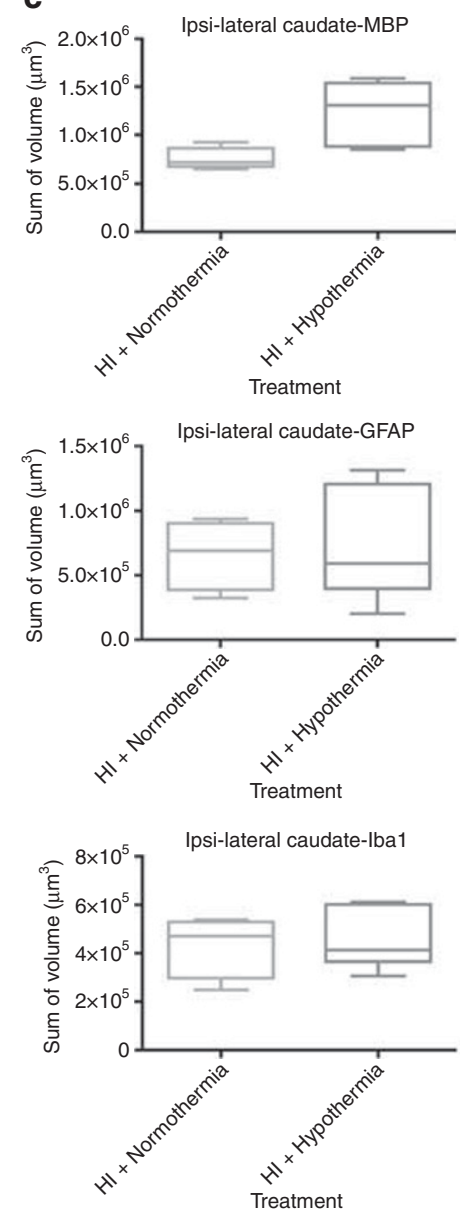

Figure 3. MBP, GFAP, and Iba1 coverage of the lateral caudate with the corpus callosum. MBP (green), GFAP (far red), and Iba1 (red). Column (a) contains samples treated with $\mathrm{HI}+$ normothermia. Column (b) contains samples treated with HI+hypothermia. (c) Volumetric measurements normalized using the ratio of intact ipsilateral tissue to intact contralateral hemisphere tissue. Data comparing $\mathrm{HI}$ treated with normothermia and $\mathrm{HI}$ treated with hypothermia using a box-and-whisker graph. The horizontal line indicates the median score. Magnification is $\times 10$. Scale bar $=100 \mu \mathrm{m}$.

groups (Figure 4c). However, qualitative imaging data again suggest that TH may play a role in the preservation of WM and an overall decrease in the features of inflammation (Figure 4a, b). The analysis of ICC sections showed decreased GFAP and Iba1 staining in the ipsilateral hippocampal regions, mostly CA2/CA3 treated with $\mathrm{TH}$ compared with normothermia (Figure 5a-c). However, the MBP staining did not show that the WM is protected in this region.

\section{Relationship Between Injury, WMI, and Inflammation}

WM and injury score correlations in TH-treated brains are suggestive of some WMI protection in the caudate (Figure 6a) when the injury score is mild $(r=-0.9077)$ when compared with normothermia-treated brains $(r=$ -0.4668). Similarly, the caudate displayed correlation between injury score and inflammation in normothermiatreated brains (Figure $\mathbf{6 b}, \mathbf{c}$ ) for GFAP $(r=0.9128)$ and Iba1 $(r=0.7524)$. Caudate hypothermia-treated correlation coefficients of GFAP $(r=0.2448)$ and Ibal $(r=0.4958)$ also displayed a positive correlation with injury score, but not as strong as the normothermia-treated correlation coefficients. The hippocampus (Figure 6d) shows a negligible difference in the correlation between WMI and injury score of normothermia- and hypothermia-treated brains.

Normothermia-treated brains displayed a strong positive correlation between injury score and inflammation in the hippocampal region (Figure 6e,f) when compared with THtreated mice. GFAP assessed in the normothermia-treated brains showed a positive correlation $(r=0.4062)$, compared with $\mathrm{TH}$ treatment, which showed a negative correlation $(r=-0.2900)$. Ibal coverage assessed in the normothermiatreated brains showed a positive correlation $(r=0.4475)$ when compared with the TH-treated brains, which showed a negative correlation $(r=-0.3770)$. In normothermia-treated brains, the degree of inflammation directly correlated with the injury score of the normothermic brains. In comparison, TH treatment displayed protection from inflammation in the hippocampal region according to the negative correlation coefficients (Figure 6e,f). 


\section{Articles | Koo et al.}

a
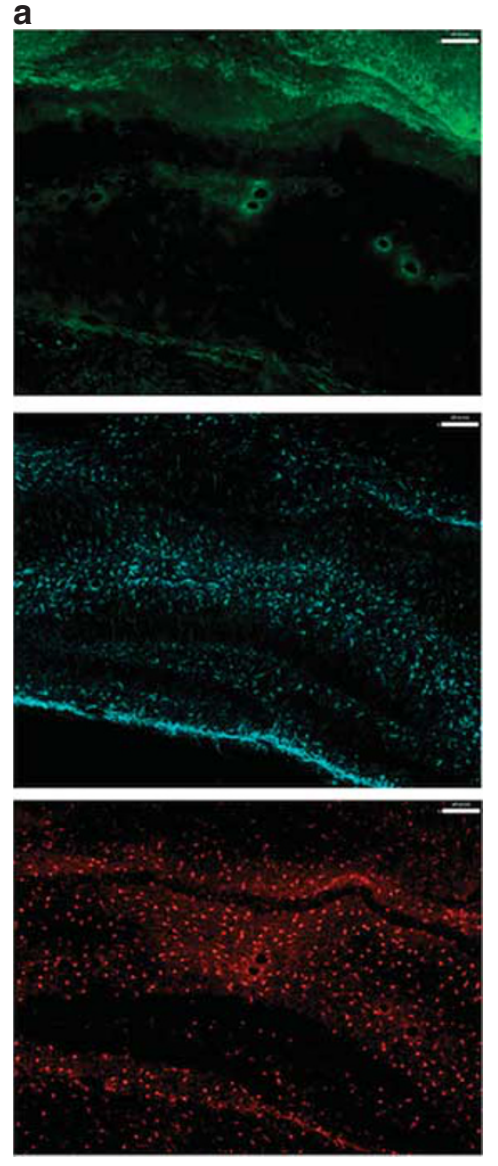

b
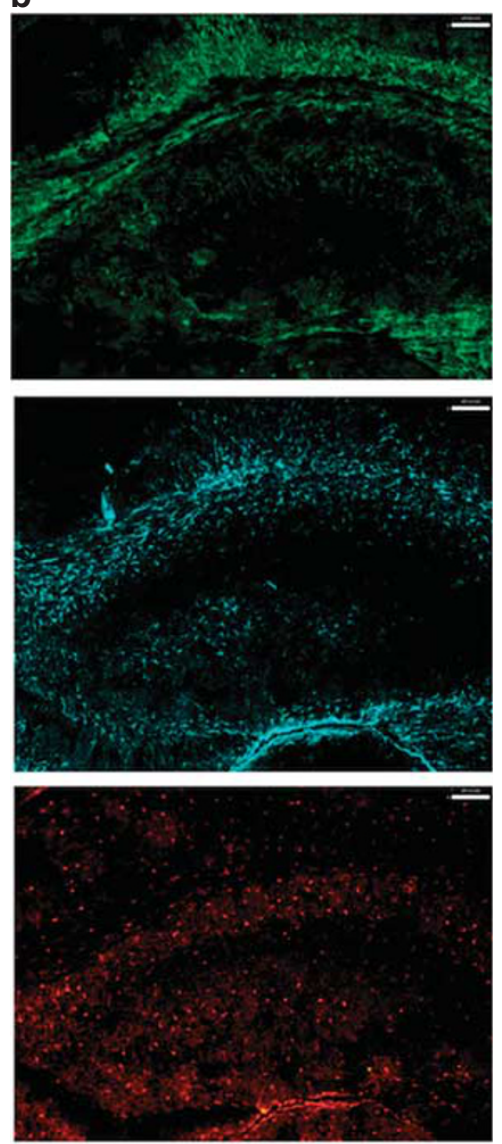

C
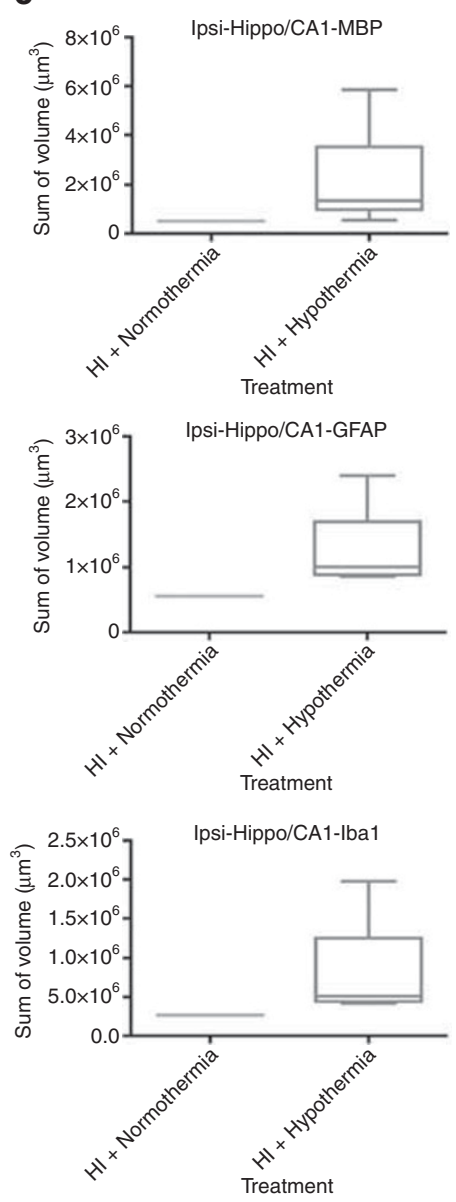

Figure 4. MBP, GFAP, and Iba1 coverage of the hippocampus CA1 with the dentate gyrus. MBP (green), GFAP (far red), and Iba1 (red). Column (a) contains samples treated with HI+normothermia. Column (b) contains samples treated with HI+hypothermia. (c) Volumetric measurements normalized using the ratio of intact ipsilateral tissue to intact contralateral hemisphere tissue. Data comparing $\mathrm{HI}$ treated with normothermia and $\mathrm{HI}$ treated with hypothermia using a box-and-whisker graph. The horizontal line indicates the median score. Magnification is $\times 10$. Scale bar $=100 \mu \mathrm{m}$.

\section{DISCUSSION}

This study establishes that $\mathrm{TH}$ in a full-term murine HI model provides overall brain protection and specifically WM protection in specific regions. The study also shows a direct correlation between the degree of $\mathrm{HI}$ injury and glial response. In addition, we demonstrate that $\mathrm{TH}$ neuroprotection is region dependent and provides protection from WMI in mildly injured brains.

WM is essential to brain connectivity and proper maturation in the neonatal brain (12). In the rodent brain, WM maturation peaks during the second-third weeks of life; thus, distorted myelination or altered composition and function of the WM after HI in P9 mice would adversely affect repair $(13,14)$. HI causes significant damage to the developing brain, including WMI. Damage and developmental impairment of WM can result in devastating outcomes such as cerebral palsy, long-term cognitive impairment, and other deficits (1). Our data in the lateral caudate region (which included a portion of corpus callosum, a region containing abundant WM) showed that $\mathrm{TH}$ provides protection from WMI. These data are consistent with previous studies that show that $\mathrm{TH}$ reduces WMI in mice and sheep $(17,18)$. However, in the caudate region, the analysis of MBP presence in relation to the degree of injury also shows that TH preserves WM from mild injury but not from severe injury.

The hippocampus is selectively vulnerable over the cortex and striatum in the HI murine model used here, and there is no significant WM protection in most hippocampal regions, but qualitative data suggest protection in penumbral regions of the hippocampus (CA2/3). The severe injury seen in this region may be resistant to the benefits of $\mathrm{TH}$ in this model $(20,21)$. These data are consistent with previous studies that demonstrated that TH-mediated attenuation of caspasedependent pathway activation after $\mathrm{HI}$ in neonatal mice is region-specific (8), and that $\mathrm{TH}$ was neuroprotective in the HI-induced penumbra, but not in the core, and that the protective effect was associated with a $\mathrm{TH}$-induced increase of antioxidant enzymes SOD-1 and GPx, leaving several inflammatory mediators unchanged, including IL1 $\beta$ and MMP-9 (22). Another possibility is that the inflammatory 
a
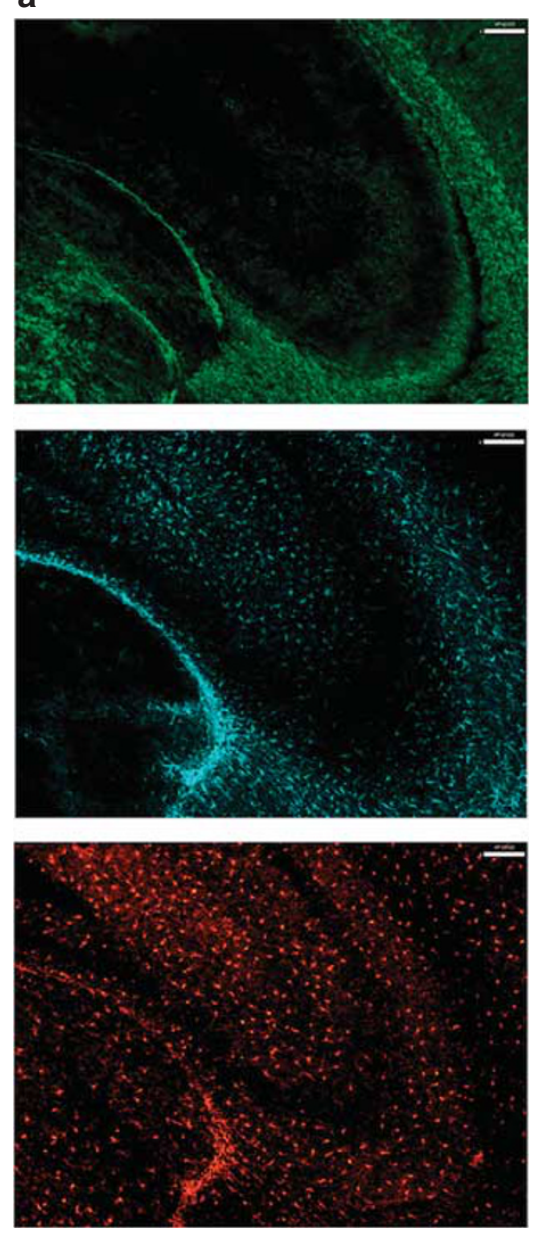

b
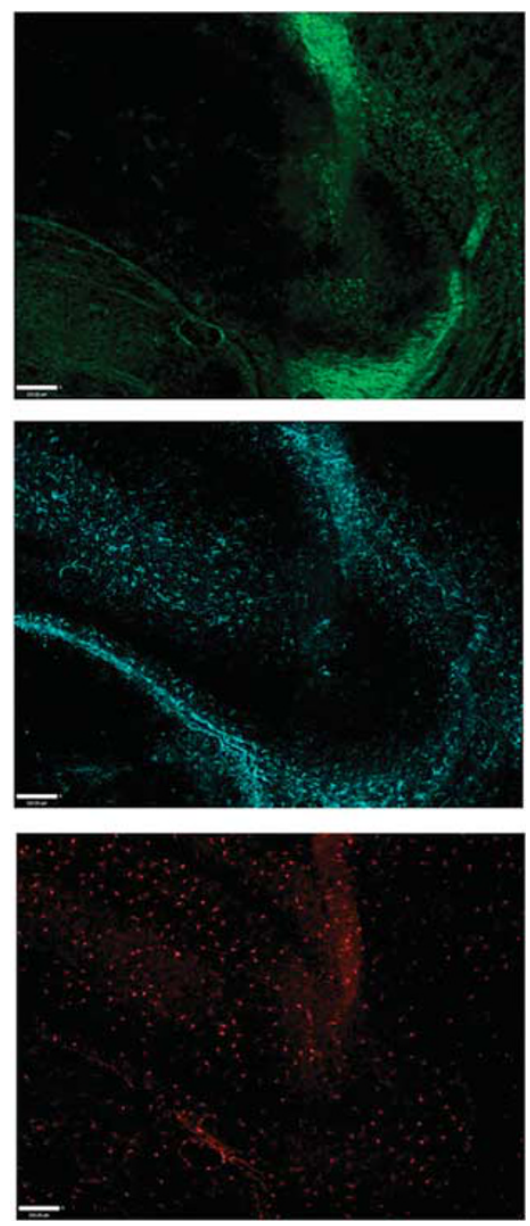

C
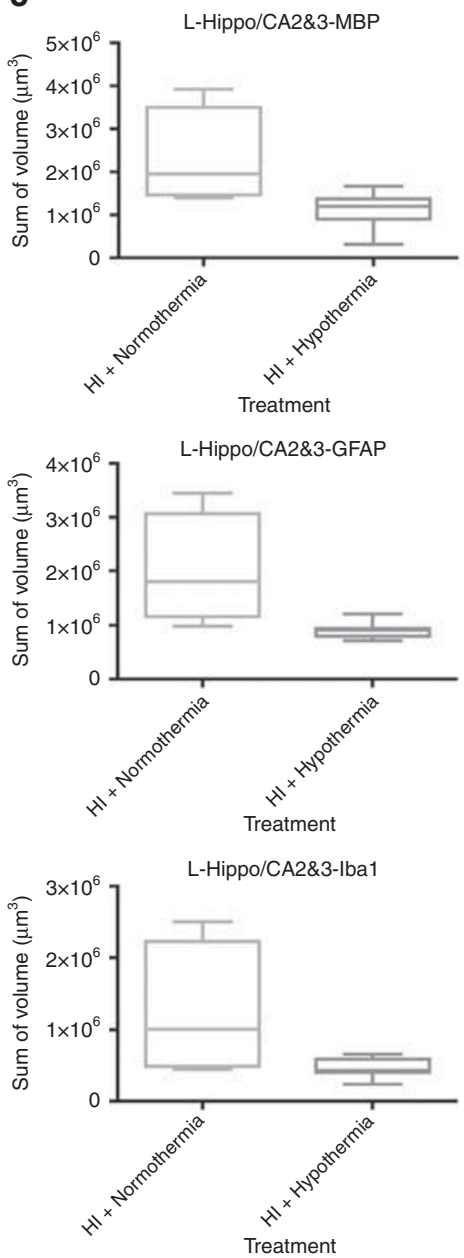

Figure 5. MBP, GFAP, and Iba1 coverage of the hippocampal regions CA2 and CA3. MBP (green), GFAP (far red), and Iba1 (red). Column (a) contains samples treated with $\mathrm{HI}+$ normothermia. Column (b) contains samples treated with HI+hypothermia. (c) Volumetric measurements normalized using the ratio of intact ipsilateral tissue to intact contralateral hemisphere tissue. Data comparing $\mathrm{HI}$ treated with normothermia and $\mathrm{HI}$ treated with hypothermia using a box-and-whisker graph. The horizontal line indicates the median score. Magnification is $\times 10$. Scale bar $=100 \mu m$.

response is strongest in the hippocampus, as evident from the Iba1 and GFAP coverage of microglia and astrocytes, respectively, thus preferentially affecting oligodendrocytes in that region (23). In addition, the hippocampus is distinct from other parenchymal regions because of its proximity to the cerebrovascular spaces and likely exposure to leukocytes in such spaces. This region has the most disturbed blood-brain barrier in the model we are using (24).

Hypothermic protection is a complex process, and there is no consensus on the best protocol to achieve beneficial effect. Several groups showed that hypothermia induced immediately after $\mathrm{HI}$ in $\mathrm{P} 7$ rats (body temperature 30 vs. $36^{\circ} \mathrm{C}$ for $10 \mathrm{~h}$ ) provided protection and inhibition of caspase activation, thereby preventing apoptotic cell death (25). Even delayed cooling of P7 rats (rectal temperature 37 vs. $30{ }^{\circ} \mathrm{C}$ for $26 \mathrm{~h}$ ) starting $2 \mathrm{~h}$ after $\mathrm{HI}$ not only significantly reduced the final size of infarction (hippocampus and, to a lesser extent, cortex) 6 weeks after the insult but also led to a long-lasting behavioral improvement throughout brain maturation despite the severity of injury (26). $\mathrm{TH}$ during the $\mathrm{HI}$ insult significantly reduced spatial learning deficits (27). On the other hand, many studies have shown that by itself hypothermia did not protect against $\mathrm{HI}$ unless it was combined with adjunctive therapies, such as topiramate (28) or phenobarbital (29). Furthermore, addition of agents such as bumetanide (a NKCC1 transporter inhibitor) augmented the neuroprotective efficacy of phenobarbital combined with hypothermia (30), demonstrating that limiting multiple pathophysiological mechanisms may be needed to provide benefits. Yet, combination of Epo with TH showed no benefit over Epo alone after HI (7). Studies in larger animals (31) have also proven that $\mathrm{TH}$ can provide neuroprotection after $\mathrm{HI}$, but, as in humans, that the severity of injury and timing, depth, and duration of hypothermia are major variables. In sheep, significant protection was achieved by initiation of hypothermia at early time points, but the protection is lost with delay for $>8 \mathrm{~h}$, and the prolongation of $\mathrm{TH}$ was not beneficial (32). 


\section{Articles | Koo et al.}
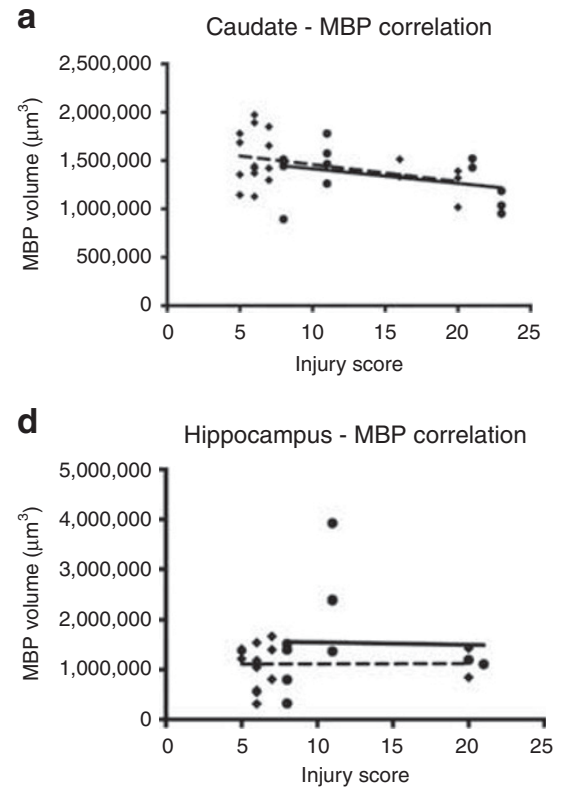

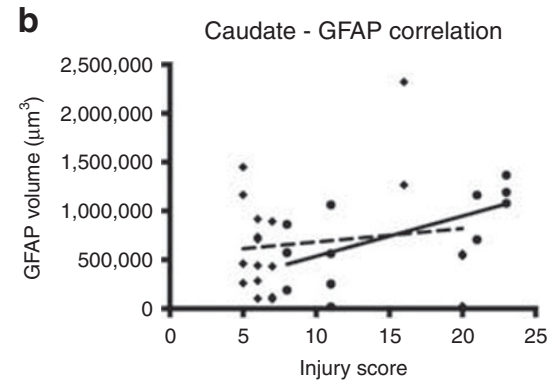

e

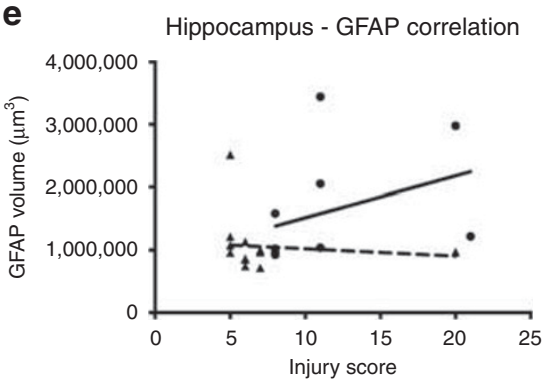

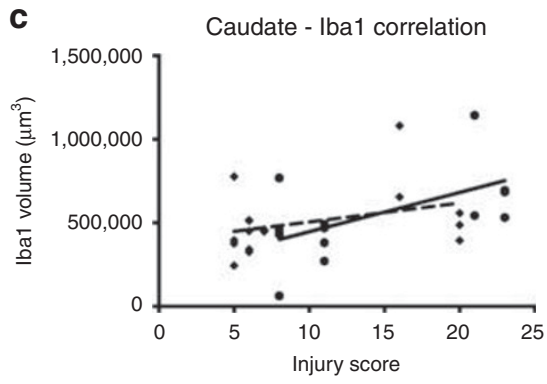

f

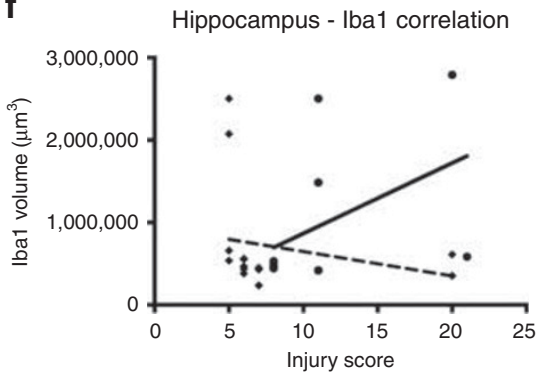

Figure 6. Correlation coefficients between injury score and volumetric raw data measurements. Normothermic (circles); hypothermic (squares). The relationship of the raw volumetric data of MBP, GFAP, and Iba1 with injury was used to determine a correlation coefficient. Caudate and hippocampal regions were evaluated. (a) MBP volumetric measurements of caudate regions compared with injury score. Normothermic, $r=-0.4668$, and hypothermic, $r=-0.9077$. (b) GFAP volumetric measurements of caudate regions compared with injury score. Normothermic, $r=0.9128$, and hypothermic, $r=0.2448$. (c) Iba1 volumetric measurements of caudate regions compared with injury score. Normothermic, $r=0.7524$, and hypothermic, $r=0.4958$. (d) MBP volumetric measurements of hippocampal regions compared with injury score. Normothermic, $r=-0.3066$, and hypothermic, $r=-0.08531$. (e) GFAP volumetric measurements of hippocampal regions compared with injury score. Normothermic, $r=0.4062$, and hypothermic, $r=-0.2900$. (f) Iba1 volumetric measurements of hippocampal regions compared with injury score. Normothermic, $r=0.4475$, and hypothermic, $r=-0.3770$.

Currently, TH is the standard care for the treatment of HI encephalopathy in neonates, but the protection of term infants is incomplete (33-36). This study shows that, in a fullterm murine model, $\mathrm{TH}$ has a significant effect in reducing the injury of mice susceptible to HI, supportive of current studies. However, the severity of injury from HI still cannot be predicted, and the variability in the degree of injury has a relationship with the protective effects of TH. Sex stratification did not show differences in our study, yet a previous study in P10 mice with HI showed that post-TH male mice showed neuroprotection, whereas the female mice had variable degrees of injury and protective effects could not be observed (17).

One limitation of this study is a variable degree of injury in individual pups. Variability in injury has been a consistent limitation noted in various murine HI studies, with severe/ extensive injury providing the greatest challenge to access $\mathrm{TH}$ protection $(4,8,17)$. Severe injury in some pups precluded us from systematically quantifying the data. The degree of damage caused by HI, along with the heterogeneity of the neuroanatomy studied, provides challenges in the analysis of these regions. Imaging modalities such as magnetic resonance imaging has been suggested, and they may provide an opportunity to better characterize and stratify the variability of injury.

HI initiates an inflammatory response, which potentiates and exacerbates neural damage (37). The presence of astrocytosis, as seen by GFAP immunolabeling, and microgliosis, as seen by Iba1 immunolabeling, are common findings in $\mathrm{HI}$ injury and support the role of neuroinflammation $(38,39)$. Our correlation analysis of the relationship between injury score and the inflammatory markers shows significant regional inflammation protection by $\mathrm{TH}$, even in the hippocampus in severely injured brains. These findings provide an opportunity for further investigation of therapy to prevent injury from $\mathrm{HI}$, as inflammatory mediators have been suggested to play a role in injury due to $\mathrm{HI}$ in the neonatal brain (40). TH with adjunctive use of anti-inflammatory mediators could possibly provide significant protection from $\mathrm{HI}$ injury. A recent review showed that numerous immunomodulatory therapies attenuating neuroinflammation show effective results in experimental models (41), supporting the use of these immunomodulating therapies with $\mathrm{TH}$ in a clinical setting of neonatal HI to prevent further neuronal injury.

Our data on the effects of TH on microglial and astroglial coverage support a relationship between the glial coverage and injury, as we observed TH-induced protection in regions with reduced glial coverage and not in regions with unaffected glial coverage. It was long believed that glial cell activation associated with $\mathrm{HI}$ is necessarily injurious, and that $\mathrm{TH}$ is protective by limiting or even aborting microglial accumulation. More recent studies in P7 rats and P9 mice subjected to focal stroke have demonstrated that microglia, in fact, contribute to endogenous protective mechanisms $(42,43)$, 
and that the microglial phenotypes, rather than microglial number, determine destructive or protective features of these cells. Combining melatonin with $\mathrm{TH}$ after $\mathrm{HI}$ in a piglet model of perinatal asphyxia protected gray matter and WM, but did not change microglial numbers; however, it affected the expression of cytotoxic molecules in microglia (44).

\section{CONCLUSION}

Our study shows that $\mathrm{TH}$ displays a significant effect in reducing the injury and inflammation in a $\mathrm{P} 9$ murine $\mathrm{HI}$ model. Protection from WMI is evident, with mildly injured brains showing the greatest benefit and overall more influential neuroprotection. Qualitative data suggest that even the penumbral hippocampal region may be protected from HI with $\mathrm{TH}$. Overall, these data indicate regional differences in WMI susceptibility and inflammation in a P9 murine HI model. Inability to score highly injured brains quantitatively and a wide range of injury was a constraint. Standardization of HI injury would provide a greater opportunity to investigate the full effects of TH regarding WMI and inflammation.

\section{SUPPLEMENTARY MATERIAL}

Supplementary material is linked to the online version of the paper at http://www.nature.com/pr

\section{ACKNOWLEDGMENTS}

EK was sponsored by the American Pediatric Society \& Society for Pediatric Research Summer Medical Student Program. This work was supported by NS 33997. DMF was supported by National Institutes of Health 33997.

Disclosure: The authors declare no conflict of interest.

\section{REFERENCES}

1. Jacobs SE, Berg M, Hunt R, Tarnow-Mordi WO, Inder TE, Davis PG. Cooling for newborns with hypoxic ischaemic encephalopathy. Cochrane Database Syst Rev 2013;1:CD003311.

2. Gonzalez FF, Ferriero DM. Therapeutics for neonatal brain injury. Pharmacol Ther 2008;120:43-53.

3. Azzopardi DV, Strohm B, Edwards AD, et al. Moderate hypothermia to treat perinatal asphyxial encephalopathy. N Engl J Med 2009;361: 1349-58.

4. Gluckman PD, Wyatt JS, Azzopardi D, et al. Selective head cooling with mild systemic hypothermia after neonatal encephalopathy: multicenter randomised trial. Lancet 2005;365:663-70.

5. Shankaran S, Laptook AR, Ehrenkranz RA, et al. Whole-body hypothermia for neonates with hypoxic-ischemic encephalopathy. N Engl J Med 2005;353:1574-84.

6. Drury PP, Gunn ER, Bennet L, Gunn AJ. Mechanisms of hypothermic neuroprotection. Clin Perinatol 2014;41:161-75.

7. Fang AY, Gonzalez FF, Sheldon RA, Ferriero DM. Effects of combination therapy using hypothermia and erythropoietin in a rat model of neonatal hypoxia-ischemia. Pediatr Res 2013;73:12-7.

8. Carlsson Y, Wang X, Schwendimann L, et al. Combined effect of hypothermia and caspase-2 gene deficiency on neonatal hypoxic-ischemic brain injury. Pediatr Res 2012;71:566-72.

9. Sabir H, Scull-Brown E, Liu X, Thoresen M. Immediate Hypothermia Is Not Neuroprotective After Severe Hypoxia-Ischemia and Is Deleterious When Delayed by 12 Hours in Neonatal Rats. Stroke 2012;43:3364-70.

10. Williams CE, Gunn AJ, Mallard C, Gluckman PD. Outcome after ischemia in the developing sheep brain: An electroencephalographic and histological study. Ann Neurol 1992;31:14-21.

11. Liu Y, Silverstein FS, Skoff R, Barks JDE. Hypoxic-ischemic oligodendroglial injury in neonatal rat brain. Pediatr Res 2002;51:25-33.
12. Nave K-A, Werner HB. Myelination of the nervous system: mechanisms and functions. Ann Rev Cell Dev Biol 2014;30:503-33.

13. McQuillen PS, Ferriero DM. Selective vulnerability in the developing central nervous system. Pediatr Neurol 2004;30:227-35.

14. Back SA, Gan X, Li Y, Rosenberg PA, Volpe JJ. Maturation-dependent vulnerability of oligodendrocytes to oxidative stress-induced death caused by glutathione depletion. J Neurosci 1998;18:6241-53.

15. Juliano C, Sosunov S, Niatsetskaya Z, et al. Mild intermittent hypoxemia in neonatal mice causes permanent neurofunctional deficit and white matter hypomyelination. Exp Neurol 2015;264:33-42.

16. Back SA, Riddle A, McClure MM. Maturation-dependent vulnerability of perinatal white matter in premature birth. Stroke 2007;38:724-30.

17. Burnsed JC, Chavez-Valdez R, Hossain MS, et al. Hypoxia-ischemia and therapeutic hypothermia in the neonatal mouse brain - a longitudinal study. PLoS ONE 2015;10:e0118889.

18. Roelfsema V, Bennet L, George S, et al. Window of opportunity of cerebral hypothermia for postischemic white matter injury in the nearterm fetal sheep. J Cereb Blood Flow Metab 2004;24:877-86.

19. Rice JE, Vannucci RC, Brierley JB. The influence of immaturity on hypoxic-ischemic brain damage in the rat. Ann Neurol 1981;9:131-41.

20. Sheldon RA, Sedik C, Ferriero DM. Strain-related brain injury in neonatal mice subjected to hypoxia-ischemia. Brain Res 1998;810: $114-22$.

21. Sheldon RA, Hall JJ, Noble LJ, Ferriero DM. Delayed cell death in neonatal mouse hippocampus from hypoxia-ischemia is neither apoptotic nor necrotic. Neurosci Lett 2001;304:165-8.

22. Chevin M, Guiraut C, Maurice-Gelinas C, Deslauriers J, Grignon S, Sébire G. Neuroprotective effects of hypothermia in inflammatory-sensitized hypoxic-ischemic encephalopathy. Int J Dev Neurosci 2016;55:1-8.

23. Graf AE, Haines KM, Pierson CR, et al. Perinatal inflammation results in decreased oligodendrocyte numbers in adulthood. Life Sci 2014;94: $164-71$.

24. Ek CJ, D’Angelo B, Lehner C, Nathanielsz P, Li C, Mallard C. Expression of tight junction proteins and transporters for xenobiotic metabolism at the blood-CSF barrier during development in the nonhuman primate $(P$. hamadryas). Reprod Toxicol 2015;56:32-44.

25. Zhu C, Wang X, Cheng X, et al. Post-ischemic hypothermia-induced tissue protection and diminished apoptosis after neonatal cerebral hypoxia-ischemia. Brain Res 2004;996:67-75.

26. Wagner BP, Nedelcu J, Martin E. Delayed postischemic hypothermia improves long-term behavioral outcome after cerebral hypoxia-ischemia in neonatal rats. Pediatr Res 2002;51:354-60.

27. Mishima K, Ikeda T, Yoshikawa T, et al. Effects of hypothermia and hyperthermia on attentional and spatial learning deficits following neonatal hypoxia-ischemic insult in rats. Behav Brain Res 2004;151: 209-17.

28. Liu Y, Barks JD, Xu G, Silverstein FS. Topiramate extends the therapeutic window for hypothermia-mediated neuroprotection after stroke in neonatal rats. Stroke 2004;35:1460-5.

29. Barks JD, Liu Y-Q, Shangguan Y, Silverstein FS. Phenobarbital augments hypothermic neuroprotection. Pediatr Res 2010;67:532-7.

30. Liu Y, Shangguan Y, Barks JDE, Silverstein FS. Bumetanide augments the neuroprotective efficacy of phenobarbital plus hypothermia in a neonatal hypoxia-ischemia model. Pediatr Res 2012;71:559-65.

31. Gunn AJ, Bennet L, Gunning MI, Gluckman PD, Gunn TR. Cerebral hypothermia is not neuroprotective when started after postischemic seizures in fetal sheep. Pediatr Res 1999;46:274-80.

32. Davidson JO, Yuill CA, Zhang FG, Wassink G, Bennet L, Gunn AJ. Extending the duration of hypothermia does not further improve white matter protection after ischemia in term-equivalent fetal sheep. Sci Rep 2016;6:25178.

33. Kattwinkel J, Perlman JM, Aziz K, et al. Neonatal resuscitation: 2010 American Heart Association Guidelines for Cardiopulmonary Resuscitation and Emergency Cardiovascular Care. Pediatrics 2010;126: e1400-13.

34. Perlman JM, Wyllie J, Kattwinkel J, et al. Part 11: neonatal resuscitation: 2010 International Consensus on Cardiopulmonary Resuscitation and 


\section{Articles | Koo et al.}

Emergency Cardiovascular Care Science With Treatment Recommendations. Circulation 2010;122:S516-38.

35. Rutherford M, Ramenghi LA, Edwards AD, et al. Assessment of brain tissue injury after moderate hypothermia in neonates with hypoxicischaemic encephalopathy: a nested substudy of a randomised controlled trial. Lancet Neurol 2010;9:39-45.

36. Shankaran S, Barnes PD, Hintz SR, et al. Brain injury following trial of hypothermia for neonatal hypoxic-ischaemic encephalopathy. Arch Dis Child Fetal Neonatal Ed 2012;97:F398-404.

37. Nathan C. Points of control in inflammation. Nature 2002;420:846-52.

38. Middeldorp J, Hol EM. GFAP in health and disease. Prog Neurobiol 2011;93:421-3.

39. McRae A, Gilland E, Bona E, Hagberg H. Microglia activation after neonatal hypoxic-ischemia. Brain Res Dev Brain Res 1995;84:245-52.
40. Saliba E, Henrot A. Inflammatory mediators and neonatal brain damage. Neonatology 2001;79:224-7.

41. Hagberg H, Mallard C, Ferriero DM, et al. The role of inflammation in perinatal brain injury. Nat Rev Neurol 2015;11:192-208.

42. Fernández-López D, Faustino J, Klibanov AL, et al. Microglial cells prevent hemorrhage in neonatal focal arterial stroke. J Neurosci 2016;36: 2881-93.

43. Chip S, Fernández-López D, Li F, Faustino J, Derugin N, Vexler ZS. Genetic deletion of galectin-3 enhances neuroinflammation, affects microglial activation and contributes to sub-chronic injury in experimental neonatal focal stroke. Brain Behav Immun 2016;60:270-81.

44. Robertson NJ, Faulkner S, Fleiss B, et al. Melatonin augments hypothermic neuroprotection in a perinatal asphyxia model. Brain 2013;136:90-105. 